IPMU 11-0020

TU-878

KEK-TH 1440

\title{
Number-Theory Dark Matter
}

\author{
Kazunori Nakayama $^{a}$, Fuminobu Takahashi $^{b, c}$ and Tsutomu T. Yanagida ${ }^{c, d}$ \\ ${ }^{a}$ Theory Center, KEK, 1-1 Oho, Tsukuba, Ibaraki 305-0801, Japan \\ ${ }^{b}$ Department of Physics, Tohoku University, Sendai 980-8578, Japan \\ ${ }^{c}$ Institute for the Physics and Mathematics of the Universe, University of Tokyo, \\ Kashiwa 277-8568, Japan \\ ${ }^{d}$ Department of Physics, University of Tokyo, Tokyo 113-0033, Japan
}

\begin{abstract}
We propose that the stability of dark matter is ensured by a discrete subgroup of the $\mathrm{U}(1)_{\mathrm{B}-\mathrm{L}}$ gauge symmetry, $Z_{2}(\mathrm{~B}-\mathrm{L})$. We introduce a set of chiral fermions charged under the $\mathrm{U}(1)_{\mathrm{B}-\mathrm{L}}$ in addition to the right-handed neutrinos, and require the anomaly-cancellation conditions associated with the $\mathrm{U}(1)_{\mathrm{B}-\mathrm{L}}$ gauge symmetry. We find that the possible number of fermions and their charges are tightly constrained, and that non-trivial solutions appear when at least five additional chiral fermions are introduced. The Fermat theorem in the number theory plays an important role in this argument. Focusing on one of the solutions, we show that there is indeed a good candidate for dark matter, whose stability is guaranteed by $Z_{2}(\mathrm{~B}-\mathrm{L})$.
\end{abstract}


The presence of dark matter (DM) in the Universe is clear observational evidence for the need of physics beyond the standard model (SM)1. The DM abundance has been measured with a very high accuracy as [2]

$$
\Omega_{\mathrm{DM}} h^{2}=0.1109 \pm 0.0056,
$$

where $h$ is the present Hubble parameter in units of $100 \mathrm{~km} / \mathrm{s} / \mathrm{Mpc}$. If the DM is made of as-yet-undiscovered particles, they must be electrically neutral and long-lived. In particular, their lifetime must be much longer than the present age of the Universe, and the longevity may be ensured by a symmetry such as a $Z_{2}$ symmetry2. There are, however, no exact global continuous or discrete symmetries, according to the argument on the quantum gravity by Banks and Seiberg [4. Therefore, it may well be that the symmetry is an unbroken subgroup of a gauge symmetry.

The $\mathrm{U}(1)_{\mathrm{B}-\mathrm{L}}$ gauge symmetry is a very attractive symmetry beyond the SM, since it predicts three families of right-handed neutrinos. The observed small masses of light neutrinos are naturally explained by the seesaw mechanism [5], provided that the B-L is broken at a very high energy such as the grand unified theory (GUT) scale. Interestingly, a $Z_{2}$ subgroup of the $\mathrm{U}(1)_{\mathrm{B}-\mathrm{L}}$ gauge symmetry remains unbroken in the low energy, if the $\mathrm{U}(1)_{\mathrm{B}-\mathrm{L}}$ is spontaneously broken by a Higgs field of charge 2, coupled to the right-handed neutrinos to generate the large Majorana masses. Here we define the normalization of the B-L charge so that the right-handed neutrinos carry the charge -1 . In this letter we consider a possibility that the $Z_{2}(\mathrm{~B}-\mathrm{L})$ is responsible for the stability of DM.

The anomaly-free conditions associated with the $\mathrm{U}(1)_{\mathrm{B}-\mathrm{L}}$ symmetry are satisfied for the SM fermions plus the three right-handed neutrinos, which however contain no DM candidate 3 . Accordingly, we need to introduce an extra particle, which must be electrically neutral and (quasi-)stable to be a good candidate for DM. In this letter we consider a set of SM-gauge-singlet chiral fermions charged under the $\mathrm{U}(1)_{\mathrm{B}-\mathrm{L}}$, some of which are to be the DM. One important constraint on those fermions is that they must satisfy the

\footnotetext{
${ }^{1}$ One exception is a primordial black hole [1, which is the only candidate for DM in the SM.

${ }^{2}$ Another possibility is a composite dark matter scenario [3].

${ }^{3}$ If one of the right-handed neutrinos is extremely light, it can account for DM. In fact, it is possible to split the mass scales of the right-handed neutrinos while keeping the success of the seesaw mechanism in the the extra dimensional framework [6].
} 
anomaly-free conditions associated with the $\mathrm{U}(1)_{\mathrm{B}-\mathrm{L}}$, which severely limit the possible number of fermions as well as their (relative) B-L charges. We assume the B-L charges to be rational, since otherwise we would have an exact global symmetry in contradiction with the quantum gravity [4]. In particular, by observing that all the SM fermions and the right-handed neutrinos have odd B-L charges, the lightest new fermion with even B-L charge will be stable and therefore a candidate for DM 5 Also, if the B-L charge is relatively large, the fermion mass tends to be light enough to be produced in the early Universe.

In this letter, we show that one of the "minimal sets" of new chiral fermions contains those with large B-L charges. Interestingly, one of them turns out to be a good candidate for the DM in the Universe. Here we define the minimal set of fermions such that it contains no two different chiral fermions which have the same B-L charge: i.e. the number of the $Q_{i}$ charged chiral fermions $\psi_{i}$ is one or zero. Thus, this is one plausible example in which the stability of DM is ensured by a discrete subgroup of a local symmetry 6

Let us first see how the anomaly-free conditions limit the possible number and charges of the additional fermions 4$]$ There are two anomaly-free conditions: one is from $\left[U(1)_{\mathrm{B}-\mathrm{L}}\right]^{3}$ anomaly and the other from the gravitational $\left[U(1)_{\mathrm{B}-\mathrm{L}}\right] \times[\text { graviton }]^{2}$ anomaly. They are given by

$$
\begin{aligned}
& \sum_{i=1}^{n} Q_{i}^{3}=0, \\
& \sum_{i=1}^{n} Q_{i}=0,
\end{aligned}
$$

where we assume $Q_{i} \neq 0$, and $n$ is the number of newly introduced fermions, $\psi_{i}(i=1$ $n)$. We define all the new chiral fermions to be right-handed fermions without loss of generality. According to the argument of Banks and Seiberg [4, $Q_{i}$ must be a rational

\footnotetext{
${ }^{4}$ The B-L corresponds to the fiveness $\mathrm{U}(1)$ in the SU(5) GUT. Notice that all the SU(5)-invariant operators of quarks and leptons $\left(5^{*}, \mathbf{1 0}\right)$ carry integer charges of the fiveness $\mathrm{U}(1)$.

${ }^{5}$ This applies to the case that the B-L charges of the additional new fermions are integer.

${ }^{6}$ Other models can be found in Ref. [7], in which the stability of DM is ensured by a discrete symmetry, a remnant of hidden $\mathrm{U}(1)$ gauge symmetry.

${ }^{7}$ Mathematical analysis for finding a set of chiral fermions satisfying the anomaly-free conditions was given in Ref. [8.
} 
number. By multiplying a non-zero integer $a$ with the both sides of Eqs. (2) and (3) , therefore, we can always rewrite them in the following form,

$$
\begin{gathered}
\sum_{i=1}^{n}\left(Z_{i}\right)^{3}=0 \\
\sum_{i=1}^{n} Z_{i}=0
\end{gathered}
$$

where $\left\{Z_{1}, \cdots Z_{n}\right\}=\left\{a Q_{1}, \cdots, a Q_{n}\right\}$ are integers. For convenience, we rearrange $\left\{Z_{i}\right\}$ in ascending order: $Z_{1}<Z_{2}<\cdots<Z_{n}$. Let us seek solutions of Eqs. (4) and (5) with $n>1$. The solutions of Eqs. (21) and (3) can be obtained by multiplying the integer solutions by some rational number.

In the case of two new fermions $(n=2)$, one can easily find that the solutions of Eqs. (4) and (5) are given by $Z_{1}=-Z_{2}$. In this case we can construct a mass term,

$$
\mathcal{L}=\frac{1}{2} m \psi_{1} \psi_{2}+\text { h.c. }
$$

The mass parameter $m$ is not constrained by any symmetry and hence can be as large as the Planck scale. This does not lead to interesting phenomenology within our framework, and hence we do not consider the case of $n=2$. In the following we will exclude vector-like fermions from the solutions.

In the case of three new fermions $(n=3)$, we can easily see that there is no solution to Eq. (4), by noting the famous Fermat theorem in the number theory [9] 8 This fact requires at least four new fermions to cancel the anomalies. Thus we already suspect that such a solution, if it exists, may contain chiral fermions of large B-L charges.

In the case of four new fermions $(n=4)$, it is easy to prove there is no phenomenologically interesting solution to the both conditions, (41) and (5). If we erase $Z_{4}$ from the two equations, we find

$$
\left(Z_{1}+Z_{2}\right)\left(Z_{2}+Z_{3}\right)\left(Z_{3}+Z_{1}\right)=0 .
$$

\footnotetext{
${ }^{8}$ Euler discovered a proof of the Fermat theorem in the special case of the cubic equation (i.e. Eq. (4) with $n=3$ ) [10, which however contained an important missing step. The complete proof was given by Kausler [1] and many others.
} 


\begin{tabular}{|c|c|c|c|c|}
\hline$Z_{1}$ & $Z_{2}$ & $Z_{3}$ & $Z_{4}$ & $Z_{5}$ \\
\hline-9 & -5 & -1 & 7 & 8 \\
\hline-9 & -7 & 2 & 4 & 10 \\
\hline-18 & -17 & 1 & 14 & 20 \\
\hline-21 & -12 & 5 & 6 & 22 \\
\hline-25 & -8 & -7 & 18 & 22 \\
\hline
\end{tabular}

Table 1: Independent solutions to Eqs. (41) and (15) for $\max \left\{\left|Z_{i}\right|\right\} \leq 25$ for $n=5$.

Thus, two of $\psi_{1}, \psi_{2}$ and $\psi_{3}$ must have the charges of opposite sign and equal magnitude, and so do the other fermion and $\psi_{4}$. The solution is therefore given by two pairs of vector-like fermions, in which we are not interested, as in the case of $n=2$.

Thus we are led to consider the case of at least five new chiral fermions $(n \geq 5)$. Introducing five additional fermions $(n=5)$ is the minimal non-trivial extension. The independent solutions to Eqs. (4) and (5) with $n=5$ and $\max \left\{\left|Z_{i}\right|\right\} \leq 25$ are given in Table 1. We find that even this minimal extension leads to relatively large B-L charges. We show in the following that the minimal charge solution in Table 1 contains a good candidate for the DM.

The seesaw mechanism [5] for neutrino mass generation suggests the Majorana mass of the (heaviest) right-handed neutrino at about the GUT scale. For this purpose, we introduce a Higgs field $\Phi$ with the B-L charge 2. We assume that $\Phi$ develops a vacuum expectation value (vev), $\langle\Phi\rangle=v_{\mathrm{B}-\mathrm{L}}$, where $v_{\mathrm{B}-\mathrm{L}}$ represents the $\mathrm{B}-\mathrm{L}$ breaking scale. The three right-handed neutrinos, $N_{1}, N_{2}$ and $N_{3}$, acquire a mass from

$$
\frac{1}{2} \kappa_{3} \Phi N_{3} N_{3}+\frac{1}{2} \kappa_{2} \Phi N_{2} N_{2}+\frac{1}{2} \kappa_{1} \Phi N_{1} N_{1}
$$

where $\kappa_{1,2,3}$ are coupling constants with $\left|\kappa_{3}\right| \geq\left|\kappa_{2}\right| \geq\left|\kappa_{1}\right|$, and we have adopted a basis such that the right-handed neutrino mass matrix is diagonalized. The breaking scale can be inferred from the neutrino oscillation data, assuming that the couplings of the heaviest right-handed neutrino are of order unity. Then the B-L breaking scale $v_{\mathrm{B}-\mathrm{L}}$ is estimated to be about $10^{15} \mathrm{GeV}$. As noted before, there is unbroken $Z_{2}(\mathrm{~B}-\mathrm{L})$, as long as the $\mathrm{U}(1)_{\mathrm{B}-\mathrm{L}}$ symmetry is broken only by the vev of $\Phi$. Since $Z_{2}(\mathrm{~B}-\mathrm{L})$ is a subgroup of the gauge symmetry, it can be an exact symmetry which ensures the stability of DM.

First, let us focus on the integer solutions to Eqs. (21) and (3), which are obtained by 
identifying $Z_{i}$ with $\pm Q_{i}$, i.e. by setting $a= \pm 1$. To be explicit we consider one of the integer solutions,

$$
\left(Q_{1}, Q_{2}, Q_{3}, Q_{4}, Q_{5}\right)=(-9,-5,-1,7,8)
$$

which corresponds to the first solution in Table 1 with $a=1$. In this example, the chiral fermion with a charge $8, \psi_{5}$, is stable because this is the only chiral fermion with an even B-L charge and it has no mixings with the other fermions. In other words, the stability of $\psi_{5}$ is guaranteed by the $Z_{2}(\mathrm{~B}-\mathrm{L})$. Therefore the fermion $\psi_{5}$ is a prime candidate for $\mathrm{DM}$ in this solution.

The $\psi_{5}$ acquires a mass from the following non-renormalizable operator,

$$
\frac{\Phi^{* 8}}{M^{7}} \psi_{5} \psi_{5}
$$

where $M$ is a cut-off scale. Then the mass of $\psi_{5}$ is given by

$$
m_{\psi_{5}} \approx 10 \mathrm{keV}\left(\frac{v_{\mathrm{B}-\mathrm{L}}}{3 \times 10^{15} \mathrm{GeV}}\right)^{8}
$$

where we have set the cut-off scale $M$ to be the Planck mass, $M_{P} \approx 2.4 \times 10^{18} \mathrm{GeV}$. In the following we will take $v_{\mathrm{B}-\mathrm{L}}=3 \times 10^{15} \mathrm{GeV}$ and $M=M_{P}$ as reference values unless otherwise stated. The mass of $\psi_{5}$ is sensitive to the B-L breaking scale, but it can be heavy enough to be consistent with the Lyman-alpha data [12].

In order to account for the DM, $\psi_{5}$ must have the correct abundance. The main production process is pair production of $\psi_{5}$ from the SM fermions in plasma through the s-channel exchange of the B-L gauge boson [6]. The production is most efficient at reheating. The $\psi_{5}$ number to entropy ratio can be roughly estimated as

$$
\begin{aligned}
Y_{\psi_{5}} \equiv \frac{n_{\psi_{5}}}{s} & \left.\sim \frac{\langle\sigma v\rangle n_{f}^{2} / H}{\frac{2 \pi^{2}}{45} g_{*} T^{3}}\right|_{T=T_{R}} \\
& \sim 4 \times 10^{-5}\left(\frac{g_{*}}{10^{2}}\right)^{-\frac{3}{2}}\left(\frac{Q_{5}}{8}\right)^{2}\left(\frac{v_{\mathrm{B}-\mathrm{L}}}{3 \times 10^{15} \mathrm{GeV}}\right)^{-4}\left(\frac{T_{R}}{4 \times 10^{13} \mathrm{GeV}}\right)^{3}
\end{aligned}
$$

where $H$ is the Hubble parameter, $g_{*}$ counts the relativistic degrees of freedom at the reheating, $\langle\sigma v\rangle \sim T^{2} / v_{\mathrm{B}-\mathrm{L}}^{4}$ is the production cross section, $n_{f} \sim T^{3}$ is the number density of the SM fermions in plasma, $T_{R}$ is the reheating temperature and the first equality is 
evaluated at the reheating. The numerical solution of the Boltzmann equation gives a consistent result [13]. The DM abundance is given by

$$
\Omega_{\psi_{5}} h^{2} \approx 0.1\left(\frac{m_{\psi_{5}}}{10 \mathrm{keV}}\right)\left(\frac{Y_{\psi_{5}}}{4 \times 10^{-5}}\right)
$$

Thus, the reheating temperature as high as $O\left(10^{13}\right) \mathrm{GeV}$ is needed to account for the DM density by this production process. The thermal leptogenesis [14] works with such a high temperature.

Let us comment on the properties of the other fermions, $\psi_{1}, \psi_{2}, \psi_{3}$, and $\psi_{4}$, which have charges of $-9,-5,-1$ and 7 , respectively. We fist note that $\psi_{3}(-1)$ will have a Majorana mass of order $v_{\mathrm{B}-\mathrm{L}}$ similarly to the right-handed neutrinos. We can also see that $\psi_{4}$ and one linear combination of $\psi_{1}$ and $\psi_{2}$ acquire a mass of order $v_{\mathrm{B}-\mathrm{L}}$ from the mass terms, $\Phi \psi_{1} \psi_{4}$ and $\Phi^{*} \psi_{2} \psi_{4}$; these heavy modes do not significantly contribute to the light neutrino masses, and so they can be safely integrated out. Also they do not have any cosmological effects, since they are not produced in the early Universe if the reheating temperature is smaller than their masses. On the other hand, the other orthogonal combination of $\psi_{1}$ and $\psi_{2}$, denoted by $\psi_{\ell}$ in the following, remains light. The $\psi_{\ell}$ obtains a mass of order $v_{\mathrm{B}-\mathrm{L}}^{5} / M^{4} \sim 7 \mathrm{TeV}$ both from the Majorana mass term and from the mixing with the right-handed neutrinos. The $\psi_{\ell}$ quickly decays into a lepton and a Higgs boson before the big bang nucleosynthesis, and therefore has no drastic effects on cosmology 9

Let us comment on a possible problem with other solutions. For instance, we consider the first solution in Table 1 with $a=-1$. Similarly, we would then have a light fermion $\psi_{\ell}^{\prime}$. Problem is that $\psi_{\ell}^{\prime}$ would generally have larger mixings with the right-handed neutrinos, which spoils the seesaw formula. Thus, for such solutions, dangerous mixings between $\psi_{\ell}^{\prime}$ and the right-handed neutrinos must be suppressed by introducing an approximate matter parity or extra dimensional set-up, in order to retain the seesaw mechanism.

Next let us consider a rational number solution to Eqs. (2) and (3). One advantage of rational number solutions is that possible dangerous mixings with the right-handed neutrinos can be suppressed. On the other hand, the stability of the lightest $Z_{2}(\mathrm{~B}-\mathrm{L})$ even fermion is not necessarily ensured because of the presence of fermions of fractional

\footnotetext{
${ }^{9}$ The lepton asymmetry produced by the right-handed neutrino decay is not washed out if the mixing of $\psi_{\ell}$ with the right-handed neutrinos is suppressed by $O(0.1)$.
} 
charge. Let us consider one example. Multiplying the previous solution with $1 / 2$, we obtain

$$
\left(Q_{1}, Q_{2}, Q_{3}, Q_{4}, Q_{5}\right)=\left(-\frac{9}{2},-\frac{5}{2},-\frac{1}{2}, \frac{7}{2}, 4\right) .
$$

As before, $\psi_{5}$ is a candidate for DM and it has a mass of $v_{\mathrm{B}-\mathrm{L}}^{4} / M^{3} \sim 6 \times 10^{6} \mathrm{GeV}$ for the same reference values of $v_{\mathrm{B}-\mathrm{L}}$ and $M$ as those in the previous case. The correct abundance is realized if the reheating temperature is $T_{R} \sim 8 \times 10^{9} \mathrm{GeV} 10$ The thermal leptogenesis works for such high $T_{R}$. The other additional fermions have fractional charges and we can see that they remain massless and their contributions to the extra radiation will be negligibly small. Moreover, since they have fractional charges, they do not mix with the SM fermion and the right-handed neutrinos. We note here that $\psi_{5}$ is not absolutely stable, because of the presence of fermions of fractional charge. In fact it mainly decays into $\psi_{2}, \psi_{3}$, the SM lepton and the Higgs boson with a lifetime more than twenty orders of magnitude longer than the present age of the Universe, for the reference values of $v_{\mathrm{B}-\mathrm{L}}$ and $M$. The lifetime is proportional to high powers of $v_{\mathrm{B}-\mathrm{L}}$, and so, the decay could contribute to the high energy cosmic-ray spectrum for a slightly large value of $v_{\mathrm{B}-\mathrm{L}}$. Note that another solution can be obtained by multiplying (14) with an odd integer, which however leads to too light DM mass.

Finally we briefly discuss other solutions. Let us focus on the integer solution of $\left(Q_{1}, Q_{2}, Q_{3}, Q_{4}, Q_{5}\right)=(-18,-17,1,14,20)$. There are three $Z_{2}(\mathrm{~B}-\mathrm{L})$ even fermions, one of which may be a candidate for DM. For a similar reason discussed above, one linear combination of $\psi_{4}$ and $\psi_{5}$ remains light. Its mass is however of the order of $\sim v_{\mathrm{B}-\mathrm{L}}^{14} / M^{13}$, and it is extremely light. The $Z_{2}(\mathrm{~B}-\mathrm{L})$ odd fermion, $\psi_{2}$, is also extremely light and it can be regarded as a stable particle in a cosmological time scale. Thus the light and stable particles in this solution are too light to be DM. Similar consideration leads us to conclude that additional fermions tend to be extremely light and DM candidates do not likely exist as the $\mathrm{U}(1)_{\mathrm{B}-\mathrm{L}}$ charges become larger. On the other hand, these light stable particles may constitute a part of extra radiation in the Universe. If the number of such light fermions is large enough, they may be able to explain the extra radiation of the

\footnotetext{
${ }^{10}$ Since the number-to-entropy ratio is proportional to $v_{\mathrm{B}-\mathrm{L}}^{-4}$ (see (12)), the DM relic density (13) does not depend on $v_{\mathrm{B}-\mathrm{L}}$. Thus the $\psi_{5}$ mass can be lighter if we choose a smaller value of $v_{\mathrm{B}-\mathrm{L}}$.
} 
Universe indicated by recent observations [2, 16, 17, 18].

Let us comment on other implications of our scenario. So far we have implicitly assumed that the $\mathrm{U}(1)_{\mathrm{B}-\mathrm{L}}$ symmetry is spontaneously broken during inflation and it is not restored after inflation. However, it is possible that the $\mathrm{U}(1)_{\mathrm{B}-\mathrm{L}}$ gets spontaneously broken after inflation, if the mass of $\Phi$ is light enough. Then the cosmic strings will be formed at the phase transition, which can be probed by the CMB measurement such as the Planck satellite. In general the DM abundance is modified in this case, but the estimate (12) remains valid as long as the phase transition occurs at $T \gtrsim 10^{14} \mathrm{GeV}$. Another implication of the integer solution (9) is that the DM mass is in the keV range so that it can affect the structure formation at small scales. We note that the lifetime of DM depends on the assumption that $\mathrm{U}(1)_{\mathrm{B}-\mathrm{L}}$ is spontaneously broken only by $\Phi$. If $Z_{2}(\mathrm{~B}-\mathrm{L})$ is also broken by another Higgs field of a B-L odd charge, the DM decays into the SM particle, which may produce observable signature in the indirect DM search. For instance, the DM decay produces an X-ray photon in the case of (9), while high-energy gamma-ray, anti-protons, and positrons are produced in the case of (14). In fact, even without the additional Higgs, the DM is not absolutely stable in case of (14), and the decay can contribute the cosmic rays if the B-L breaking scale is several times larger than our reference value. On the other hand, no signals are expected in the direct DM search. We have seen that a high reheating temperature is needed in order to obtain a correct DM abundance (12) in the case of (9). Since the inflaton mass must be larger than the reheating temperature [20], the chaotic inflation [21] is one of the possibilities. This may be confirmed by the observation of the B-mode in the cosmic microwave background polarization.

In this letter we have considered a possibility that the longevity of DM is guaranteed by the $Z_{2}(\mathrm{~B}-\mathrm{L})$. To this end we have introduced $n$ SM-gauge-singlet chiral fermions charged under the $\mathrm{U}(1)_{\mathrm{B}-\mathrm{L}}$, in addition to the three right-handed neutrinos. We have shown that more than four additional chiral fermions are required for the cancellation of the anomalies associated with the $\mathrm{U}(1)_{\mathrm{B}-\mathrm{L}}$. In the case of $n=2$, two fermions must have charges of opposite sign and same magnitude, and they can be integrated out if their 
mass is as heavy as the Planck scale. Importantly, the Fermat theorem excludes the case of $n=3$, which have forced us to consider $n>3$. In the case of $n=4$, the four fermions are divided into two pairs of vector-like fermions, which have charges of opposite sign and same magnitude, and this case was not of our interest. Thus, we are led to consider more than four additional chiral fermions. Interestingly, one of the minimal set of the integer B-L charges satisfying the anomaly-free conditions contains a good candidate for DM. The DM abundance can be explained if the reheating temperature is as high as $O\left(10^{13}\right) \mathrm{GeV}$ or $O\left(10^{9}\right) \mathrm{GeV}$, depending on whether the charges are integer or fractional. Both cases are consistent with the thermal leptogenesis scenario.

\section{Acknowledgment}

We would like to thank Satoshi Kondo for useful discussions and particularly for his interdisciplinary colloquium on Number Theory at IPMU, where the basic idea of the present letter occurred to us. This work was supported by the Grant-in-Aid for Scientific Research on Innovative Areas (No. 21111006) [KN and FT], Scientific Research (A) (No. 22244030 [FT] and 22244021 [TTY]), and JSPS Grant-in-Aid for Young Scientists (B) (No. 21740160) [FT]. This work was also supported by World Premier International Center Initiative (WPI Program), MEXT, Japan.

\section{References}

[1] See, for example, P. H. Frampton, M. Kawasaki, F. Takahashi and T. T. Yanagida, JCAP 1004 (2010) 023 [arXiv:1001.2308 [hep-ph]].

[2] E. Komatsu et al. [ WMAP Collaboration ], Astrophys. J. Suppl. 192, 18 (2011). arXiv:1001.4538 [astro-ph.CO]].

[3] K. Hamaguchi, S. Shirai and T. T. Yanagida, Phys. Lett. B 654 (2007) 110 arXiv:0707.2463 [hep-ph]].

[4] T. Banks and N. Seiberg, arXiv:1011.5120 [hep-th]].

[5] T. Yanagida, in Proceedings of the "Workshop on the Unified Theory and the Baryon Number in the Universe", Tsukuba, Japan, Feb. 13-14, 1979, edited by O. Sawada and 
A. Sugamoto, KEK report KEK-79-18, p. 95, and "Horizontal Symmetry And Masses Of Neutrinos" , Prog. Theor. Phys. 64 (1980) 1103; M. Gell-Mann, P. Ramond and R. Slansky, in "Supergravity" (North-Holland, Amsterdam, 1979) eds. D. Z. Freedom and P. van Nieuwenhuizen, Print-80-0576 (CERN); see also P. Minkowski, Phys. Lett. B 67, 421 (1977).

[6] A. Kusenko, F. Takahashi and T. T. Yanagida, Phys. Lett. B 693 (2010) 144 arXiv:1006.1731 [hep-ph]].

[7] B. Batell, Phys. Rev. D 83, 035006 (2011) [arXiv:1007.0045 [hep-ph]].

[8] P. Batra, B. A. Dobrescu and D. Spivak, J. Math. Phys. 47 (2006) 082301 arXiv:hep-ph/0510181; for earlier works, see e.g. S. M. Barr, B. Bednarz and C. Benesh, Phys. Rev. D 34 (1986) 235.

[9] A. Wiles, Annals of Mathematics 141, 3, 443 (1995); R. Taylor and A. Wiles, Annals of Mathematics 141, 3, 553 (1995).

[10] L. Euler, Vollständige Anleitung zur Algebra, 2 Volumes. Royal Acad. Sci., St. Petersburg, 1770.

[11] C. F. Kausler, "Nova demonstratio theorematis nec summam, nec differentiam duorum cuborum cubum esse posse". Novi Acta Acad. Petrop. 13 (1795/6), 1802, 245 253.

[12] A. Boyarsky, J. Lesgourgues, O. Ruchayskiy and M. Viel, JCAP 0905 (2009) 012 arXiv:0812.0010 [astro-ph]].

[13] S. Khalil and O. Seto, JCAP 0810, 024 (2008) [arXiv:0804.0336 [hep-ph]]; G. Gelmini, S. Palomares-Ruiz and S. Pascoli, Phys. Rev. Lett. 93, 081302 (2004) arXiv:astro-ph/0403323]; G. Gelmini, E. Osoba, S. Palomares-Ruiz and S. Pascoli, JCAP 0810, 029 (2008) [arXiv:0803.2735 [astro-ph]].

[14] M. Fukugita and T. Yanagida, Phys. Lett. B 174, 45 (1986); see, for a review, W. Buchmuller, R. D. Peccei and T. Yanagida, Ann. Rev. Nucl. Part. Sci. 55, 311 (2005) arXiv:hep-ph/0502169.

[15] A. A. Aguilar-Arevalo et al. [ The MiniBooNE Collaboration ], Phys. Rev. Lett. 105, 181801 (2010). [arXiv:1007.1150 [hep-ex]]. 
[16] Y. I. Izotov, T. X. Thuan, Astrophys. J. 710, L67-L71 (2010). arXiv:1001.4440 [astro-ph.CO]]; E. Aver, K. A. Olive, E. D. Skillman, JCAP 1005, 003 (2010). arXiv:1001.5218 [astro-ph.CO]]; E. Aver, K. A. Olive, E. D. Skillman, arXiv:1012.2385 [astro-ph.CO]].

[17] K. Nakayama, F. Takahashi and T. T. Yanagida, Phys. Lett. B 697, 275 (2011) arXiv:1010.5693 [hep-ph]].

[18] L. M. Krauss, C. Lunardini and C. Smith, arXiv:1009.4666 [hep-ph]; P. C. de Holanda, A. Y. Smirnov, arXiv:1012.5627 [hep-ph]].

[19] G. C. Branco, R. Gonzalez Felipe, F. R. Joaquim et al., Phys. Lett. B562, 265-272 (2003). hep-ph/0212341].

[20] E. W. Kolb, A. Notari, A. Riotto, Phys. Rev. D68, 123505 (2003). hep-ph/0307241;

M. Drewes, arXiv:1012.5380 [hep-th]].

[21] A. D. Linde, Phys. Lett. B 129, 177 (1983). 